\title{
Retinal Vasculitis: Correlation of Animal and Human Disease
}

\author{
M. R. STANFORD ${ }^{1}$, E. M. GRAHAM ${ }^{2}$, E. KASP ${ }^{1}$, E. C. BROWN ${ }^{1}$, D. C. DUMONDE ${ }^{1}$, \\ and M. D. SANDERS ${ }^{2}$
}

London

\begin{abstract}
Summary
A form of experimental retinal vasculitis was induced in black hooded Lister rats by the inoculation of retinal S-antigen. Comparison of this disease with retinal vasculitis in man showed striking clinical, angiographic and pathological similarities. Clinically disc oedema, periphlebitis and retinal infiltrates were observed with corresponding leakage of dye on fluorescein angiography. Pathologically the disease showed perivascular lymphocytic infiltrates with focal photoreceptor necrosis. These characteristic features make this an ideal model for the study of the pathogenesis of retinal vasculitis in man.
\end{abstract}

The aetiology, pathogenesis and classification of retinal vasculitis has provoked considerable controversy. To ophthalmologists the term encompasses a clinical syndrome which occurs predominantly in young people ${ }^{1}$ and is characterised by cellular infiltration of the vitreous, disc oedema, and periphlebitis often associated with macular oedema. It is primarily a disorder expressed in the venous circulation of the eye and may be associated with certain systemic disorders such as Behçet's disease and sarcoidosis, but usually occurs without systemic features, though serological abnormalities may exist. ${ }^{2,3}$ Other systemic vasculitides, such as systemic lupus erythematosus (SLE) and polyarteritis nodosa produce arteriolar lesions without a significant inflammatory component of the retinal vessels. ${ }^{4}$ There is evidence that antiretinal autoimmunity plays a major role in the causation of retinal vasculitis, but the precise mechanisms of tissue damage remain obscure. ${ }^{5}$ Pathologically the hallmark of this disease is perivascular cuffing by inflammatory cells of the retinal vessels associated with retinal destruction, but whether this necrosis is mediated via retinal or choroidal routes or both is unknown.

To study the mechanisms involved in the mediation of this disorder and uveitis a number of animal models have been developed. In the subhuman primate, disease very similar to retinal vasculitis in man has been produced by the inoculation of a soluble retinal protein-retinal S-antigen-in Freund's complete adjuvant. ${ }^{12}$ Other models exist, again induced by retinal S-antigen, in guineapigs ${ }^{8}$ rabbits, and albino rats. ${ }^{7}$ In the latter animal, disease occurs as a panophthalmitis in the third week post inoculation, and details of the fundus and posterior chamber are rapidly lost, so that clinical signs must be inferred from histological examination.

From Departments of Immunology ${ }^{1}$ and Medical Ophthalmology ${ }^{2}$, United Medical and Dental Schools, St Thomas' Campus, London, SE1 7EH.Correspondence to: Mr. M. R. Stanford, FRCS, Department of Ophthalmology, King's College Hospital, London SE5. 
In this study we present the clinical, angiographic and pathological features of retinal vasculitis in the black hooded Lister rat. Disease was induced by inoculation of retinal S-antigen in Freund's complete adjuvant (FCA). In this model significant anterior uveitis was not observed allowing an unrestricted view of the posterior segment and photographic documentation of the evolution of clinical signs. In this paper we have compared this disease with the clinical and pathological features of retinal vasculitis in man.

\section{Materials and Methods}

\section{Animals}

The animals used in this study were black hooded Lister rats weighing $200 \mathrm{~g}$ (Bantin and Kingman). Animals were inoculated with $50 \mu \mathrm{g}$ of porcine retinal S-antigen (approximate. purity 80 per cent) in FCA via the hind foot-pad. Retinal S-antigen was prepared by size and hydrophobic absorption chromatography as described elsewhere. ${ }^{6}$ Control animals received an equal volume of phosphate buffered saline (PBS) in FCA. Animals were examined by slip lamp biomicroscopy and a standard fundus camera (Zeiss) from day ten post inoculation on a daily basis. At the termination of each experiment, or where particular clinical features were noted, the animals were anaesthetised with intraperitoneal urethane $(1.5 \mathrm{~g} / \mathrm{Kg})$ and fluorescein angiography was carried out. The tail vein of the animal was cannulated with a 25 gauge butterfly needle that had been heparinised to ensure patency. $0.3 \mathrm{ml}$ of $5 \%$ sodium fluorescein was injected and an angiogram recorded using Ilford FP4 film and standard fluorescein filters. The tail to eye time was approximately two seconds. The angiogram was terminated at five minutes and the animal killed by cardiac puncture and cervical dislocation.

The eyes were enucleated and placed in $4 \%$ gluteraldehyde for twelve hours. They were then trimmed and placed in $10 \%$ neutral buffered formalin for a further 24 hours before being dehydrated in graded alcohols, cleared in toluene and wax embedded. Paraffin sections were taken at $100 \mu \mathrm{m}$ intervals. Haematoxylin and eosin $(\mathrm{H}$. and $\mathrm{E}$.) staining was used throughout.

\section{Experimental Groups}

The animals were divided into three main groups with weight matched controls.

Group I Ten animals were followed with serial fundus photographs until the disease had settled. There were five controls.
Group II Ten animals were inoculated and observed until early signs of disease appeared when they had fluorescein angiography and the experiment was terminated. Five controls were used.

Group III Ten animals were observed on a daily basis until their disease had settled and then on a weekly basis for a further three months to check for spontaneous recurrence of disease. Five animals were used as controls.

\section{Patients}

Illustrative clinical and angiographic photographs were taken from the files of patients attending the Medical Eye Unit, St Thomas' Hospital. Pathological specimens of eyes enucleated with a diagnosis of Eales' disease or idiopathic retinal vasculitis were examined from the collection of The Institute of Ophthalmology, London (by kind permission of Professor A.Garner).

\section{Results}

\section{Clinical Observation}

The disease was divided into three stages.

Early Stage The earliest sign observed clinically was fattening and tortuosity of the retinal veins with some cellular infiltration of the vitreous. Within two days the optic disc became swollen, details of the nerve fibre layer were lost and small intraretinal infiltrates appeared in the region of the post capillary venule.

Intermediate Stage Signs present in the early stage of disease became more pronounced. Vitreous infiltration increased though this rarely obscured fundal details. The disc became more swollen, in some cases associated with peripapillary nerve fibre haemorrhages. The intraretinal infiltrates increased and areas of distinct periphlebitis appeared. In severe cases small discrete elevated lesions were evident at the level of the retinal pigment epithelium (RPE).

Late Stage As the disease settled the disc and peripapillary area returned to normal. The retinal veins became thinned and the arteries tortuous, often showing peri-arteriolar sheathing. At the end of disease the calibre of the retinal vessels was markedly reduced compared to controls. Areas of retinal infiltration 
disappeared, in some cases leaving areas of pigment epithelial atrophy.

Significant anterior uveitis (more than 1+ cells), cataract formation, and posterior synechiae did not develop in any animal. Control animals remained normal throughout. The clinical features of the disease are summarised in Table I.

\section{Fluorescein Angiography}

The fluorescein changes related to the physical signs. Disc oedema was associated with early leakage of dye which increased in the late films (Fig. 1), and periphlebitis and retinal infiltrates by leakage of dye in the venous phase. Most animals showed marked microvascular leakage (Fig. 2a) which appeared similar to that seen in patients (Fig. 2b). When disease had settled for some time (Group III) no persistent leakage of dye was evident from the disc or in areas where there had been inflammatory infiltration.

\section{Histology}

A small mononuclear cell infiltrate was seen in the anterior segment at the height of the intermediate stage. No significant infiltration of the iris or ciliary body was seen, nor was there evidence of cataract or posterior synechiae.

In the posterior segment the earliest evidence of disease was perivascular lymphocytic infiltration of the central retinal vessels that occurred predominantly around the retinal veins. This was associated with leakage of inflammatory cells into the vitreous. Figure $3 \mathrm{a}$ shows an optic disc at high power in a rat sixteen days after inoculation and is compared with a human optic disc taken from a patient with Behçet's disease (Figure 3b). In the retina a similar infiltration was seen around retinal vessels, again more pronounced in veins (Figure 4a) and is compared to a section taken from the retinal periphery in a patient with idiopathic retinal vasculitis (Fig. 4b). Both sections show that at this stage retinal vasculitis may not be associated with photoreceptor destruction. The next development in the experimental disease was the appearance of mononuclear cells in the outer segment of the photoreceptor layer. These cells went on to form clumps and caused focal photoreceptor necrosis (Figure 5). Significant choroidal infiltration or thickening was not observed. In the late stages the areas of photoreceptor loss were replaced by glial tissue.

\section{Discussion}

The difficulties associated with unravelling the precise pathogenesis of retinal vasculitis are numerous. It is primarily a disorder of young people in whom the disease may be suppressed with the appropriate systemic therapy, and is self limiting. The number of eyes available for pathological examination are few and these usually have end stage disease so that early changes which may be related to the pathogenesis are not observed. Animal models of experimental allergic uveitis (EAU) now exist, in most of which disease is induced by a single foot-pad injection of purified retinal protein in adjuvant. ${ }^{7,8}$ The nature of the disease produced depends on the species and strain of the animal used, the origin and the dose of antigen, and the type of adjuvant. ${ }^{9}$ In most animal models the

Table I Clinical features of experimental retinal vasculitis in 30 animals inoculated with s-antigen

\begin{tabular}{llll}
\hline & & Experiments (Total 30) & Control (Total 15) \\
\hline Number of animals & Developing disease & 27 & 0 \\
& Anterior uveitis (1+ cells) & 2 & 0 \\
& Vitreous cells (1-2+) & 27 & 0 \\
& Disc oedema & 27 & 0 \\
& Retinal vascular changes & 27 & 0 \\
& Retinal infiltrates & 25 & 0 \\
& Spontaneous recurrence & & $0 / 5$ \\
Disease onset & (Group III) & 0 & 0 \\
Disease duration & & $25.1 \pm 2.2$ days & 0 \\
\hline
\end{tabular}




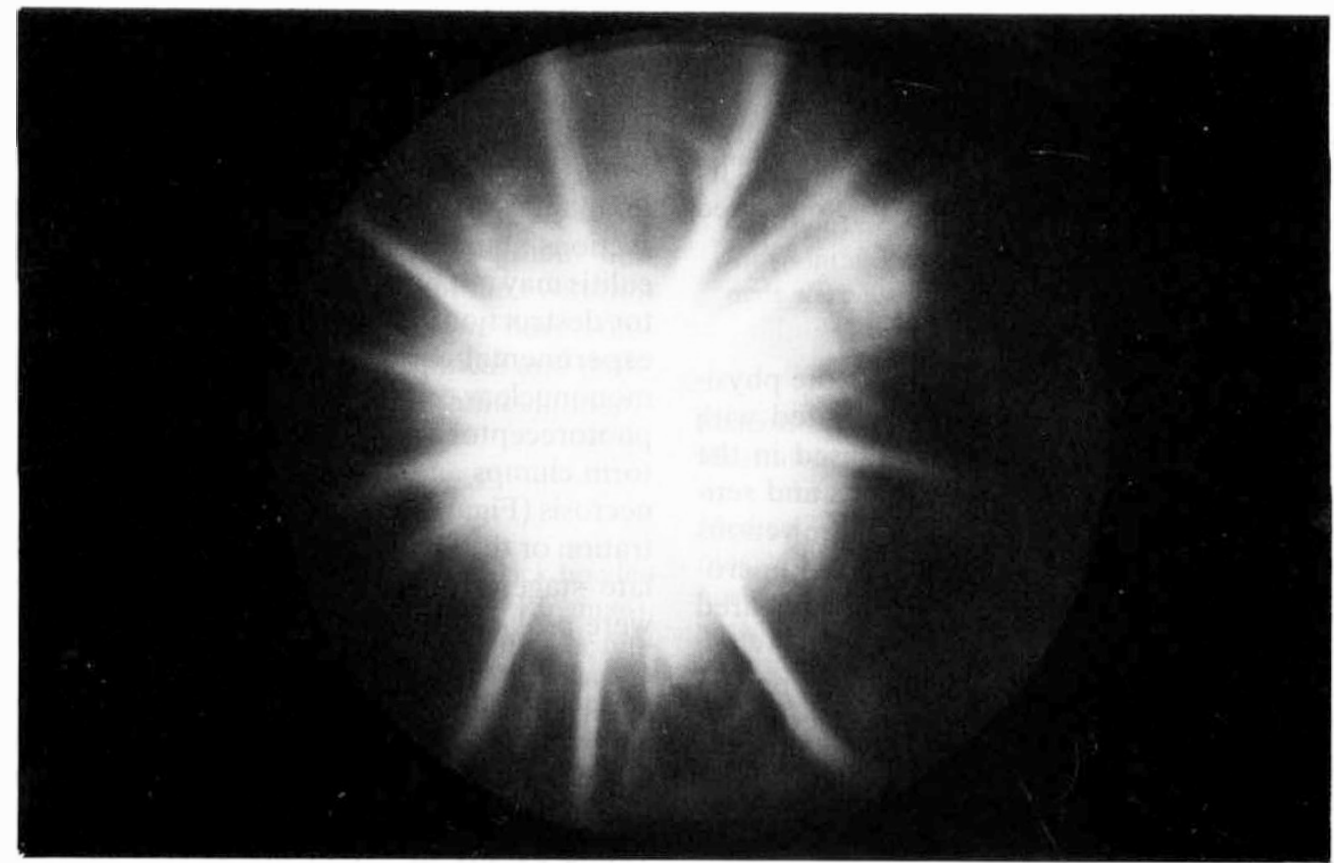

a

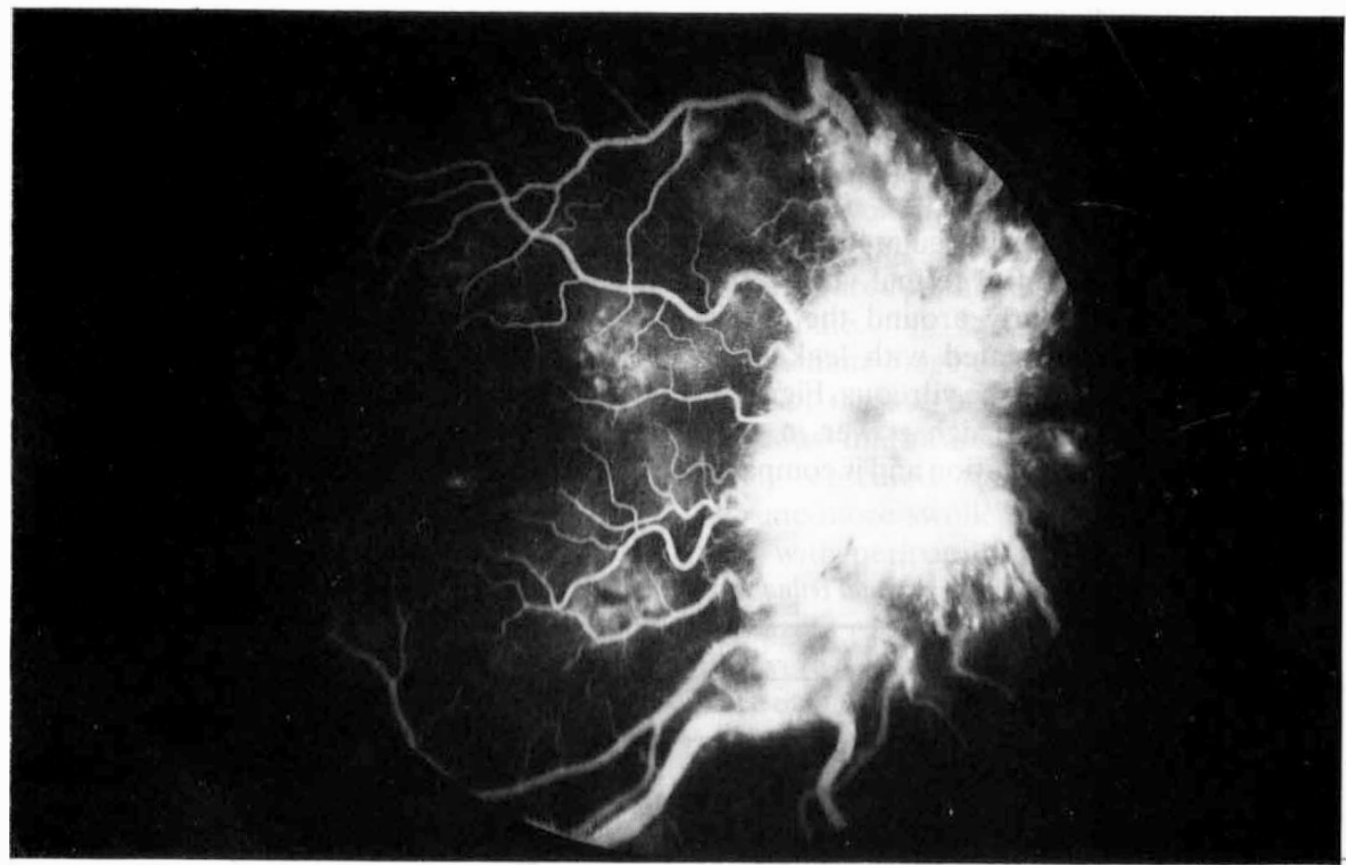

b

Fig. 1. (a) Rat: Late phase fluorescein angiogram of the disc sixteen days post inoculation showing marked leakage of dye. (b) Human: Late phase fluorescein angiogram of the disc of a 23 year old girl with active sarcoidosis showing dilated capillaries and leakage. 


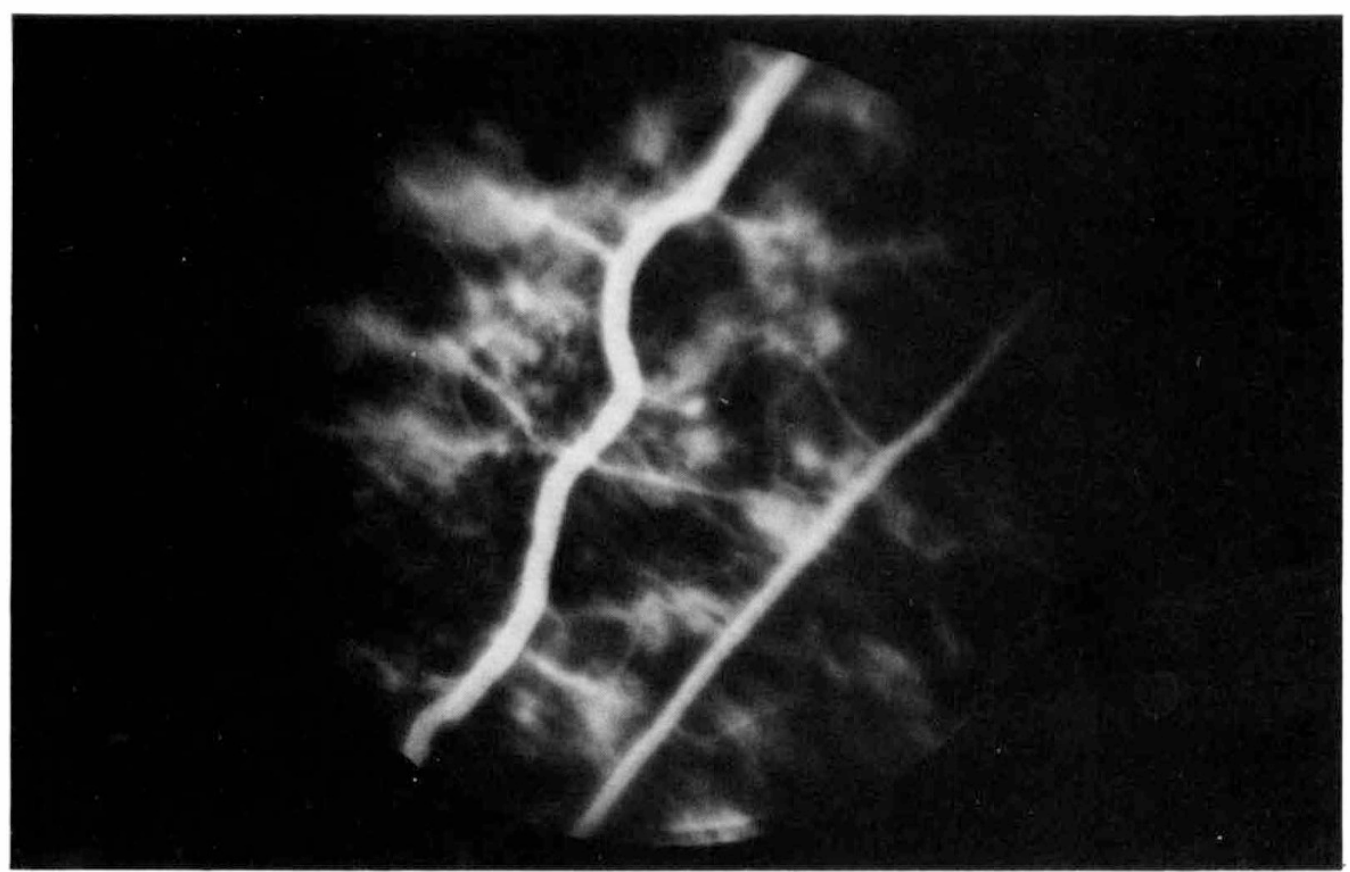

a

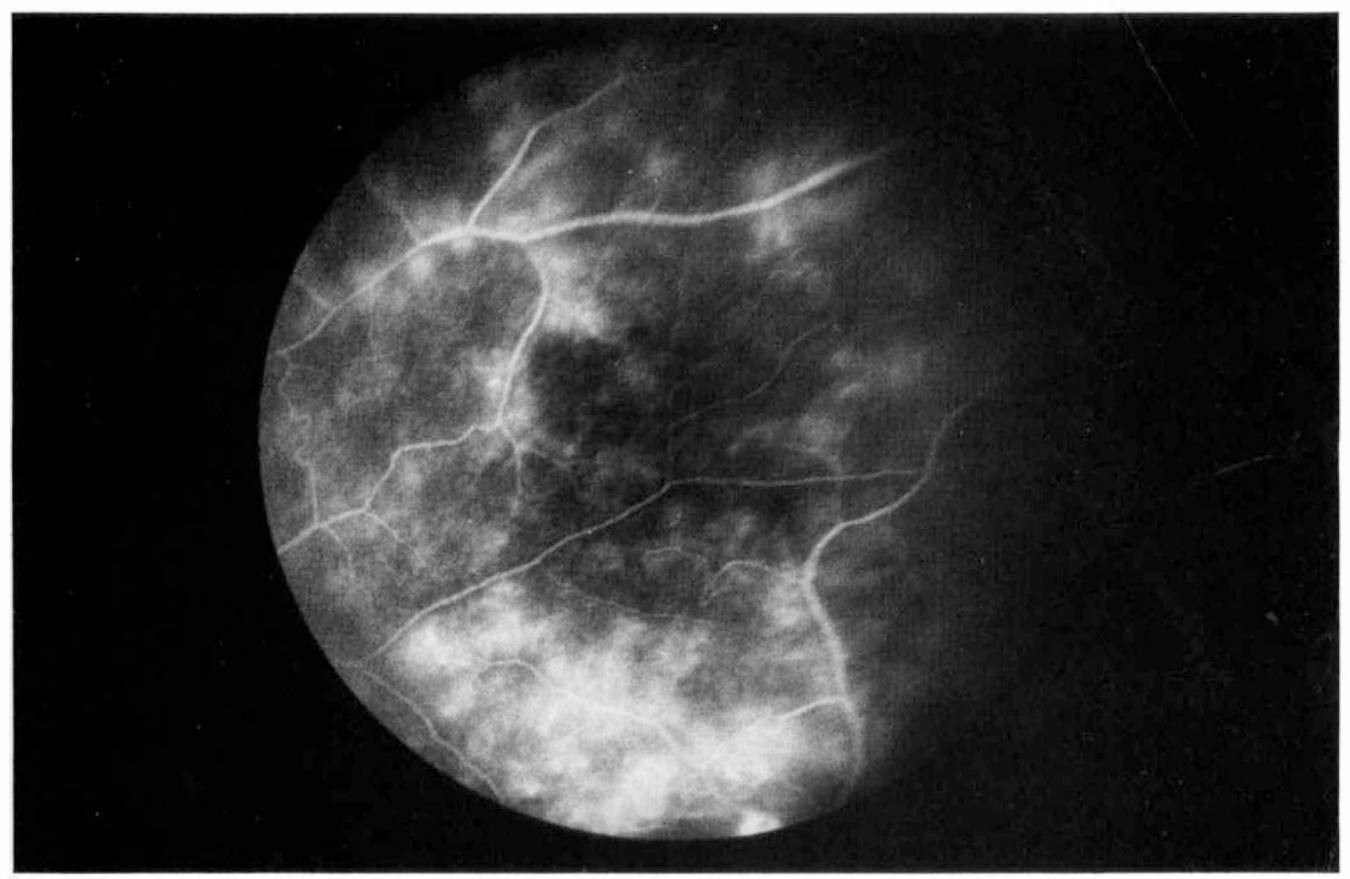

b

Fig. 2. (a) Rat: Mid venous phase angiogram of the retinal periphery (same animal as 1a) showing diffuse microvascular leakage. (b) Human: Mid venous phase angiogram of a 42 year old man with Behçet's disease showing a similar pattern of diffuse capillary leakage. 


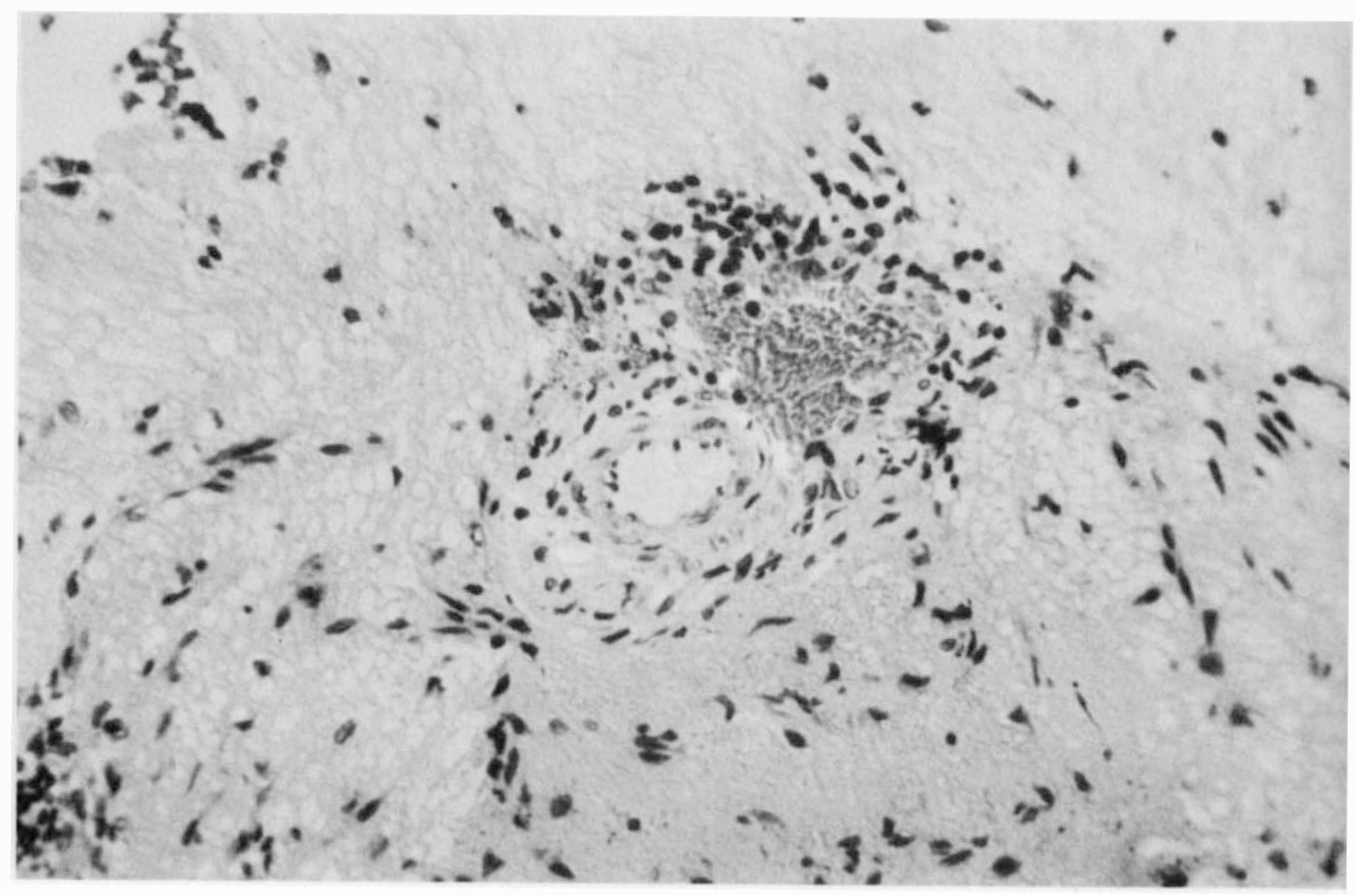

a

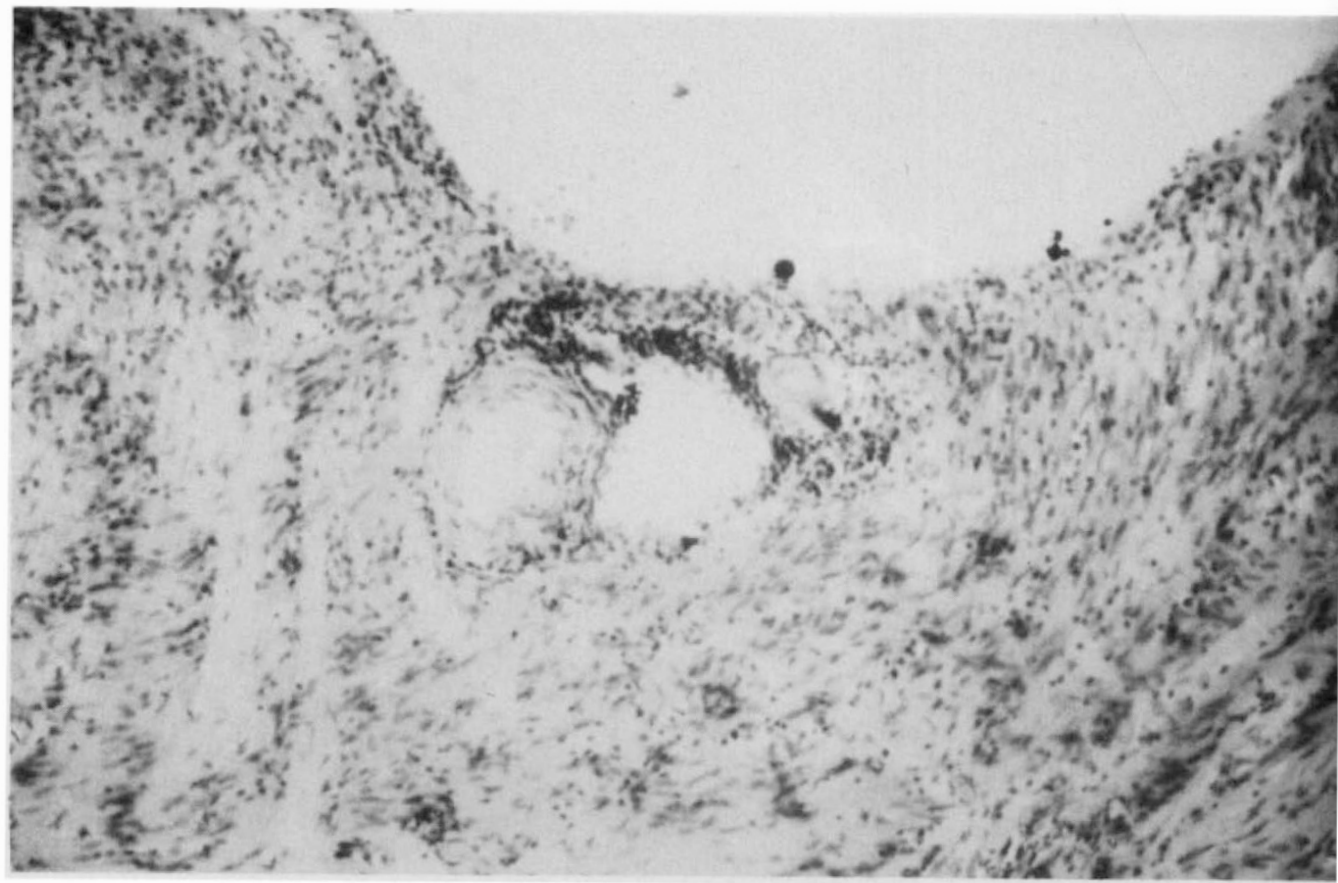

b

Fig. 3. (a) Rat: Section of optic disc showing lymphocytic infiltration of the central retinal vein $(H$. and $E$. $\times 500)$. (b) Human: Section of optic disc from the enucleated globe of a twenty three year old man with Behçet's showing active vaculitis of the central retinal vein $(H$. and $E . \times 250)$. 


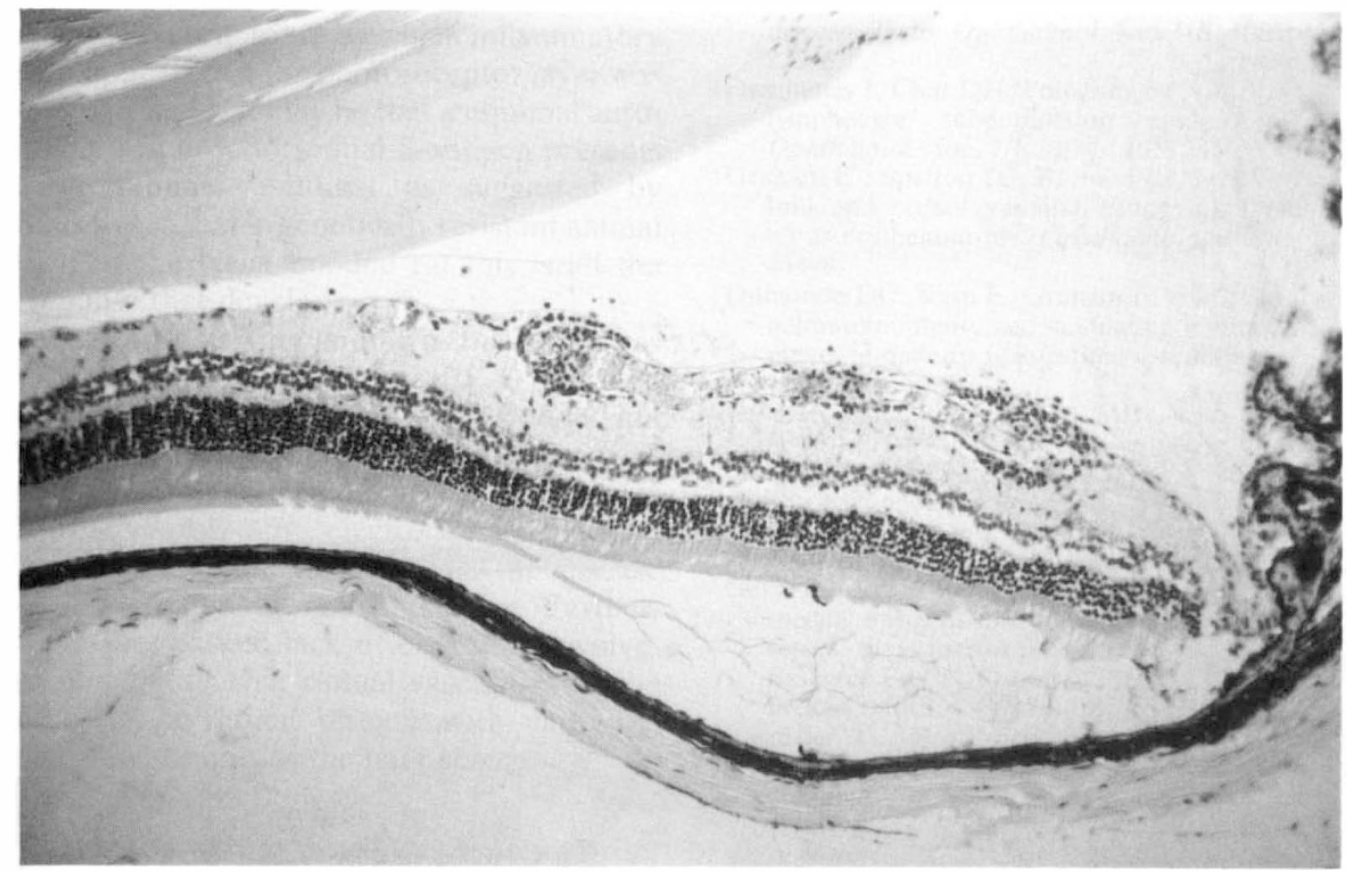

a

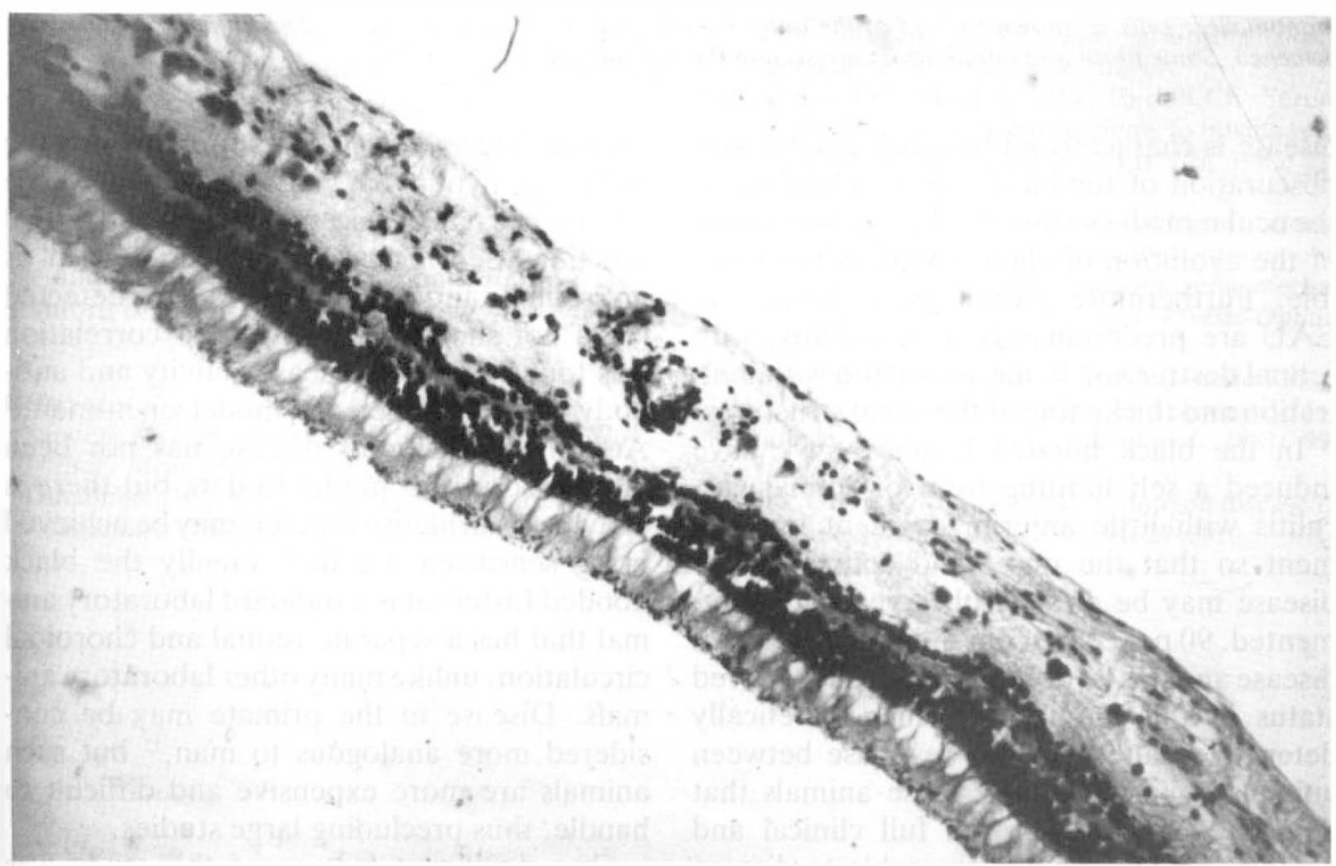

b

Fig. 4. Rat: Section of retinal periphery at eighteen days post inoculation. There is focal periphlebitis but the retina remains unchanged. No choroidal involvement is seen. There is a thin layer of subretinal fluid. The retina is artefactually detached $(H$. and E. $\times 200)$. (b) Human: Section of retinal periphery taken from the eye of a patient with idiopathic retinal vasculitis. This shows isolated vasculitis with no other change in the retina. The retina is artefactually detached $(H$. and $E . \times 125)$. 


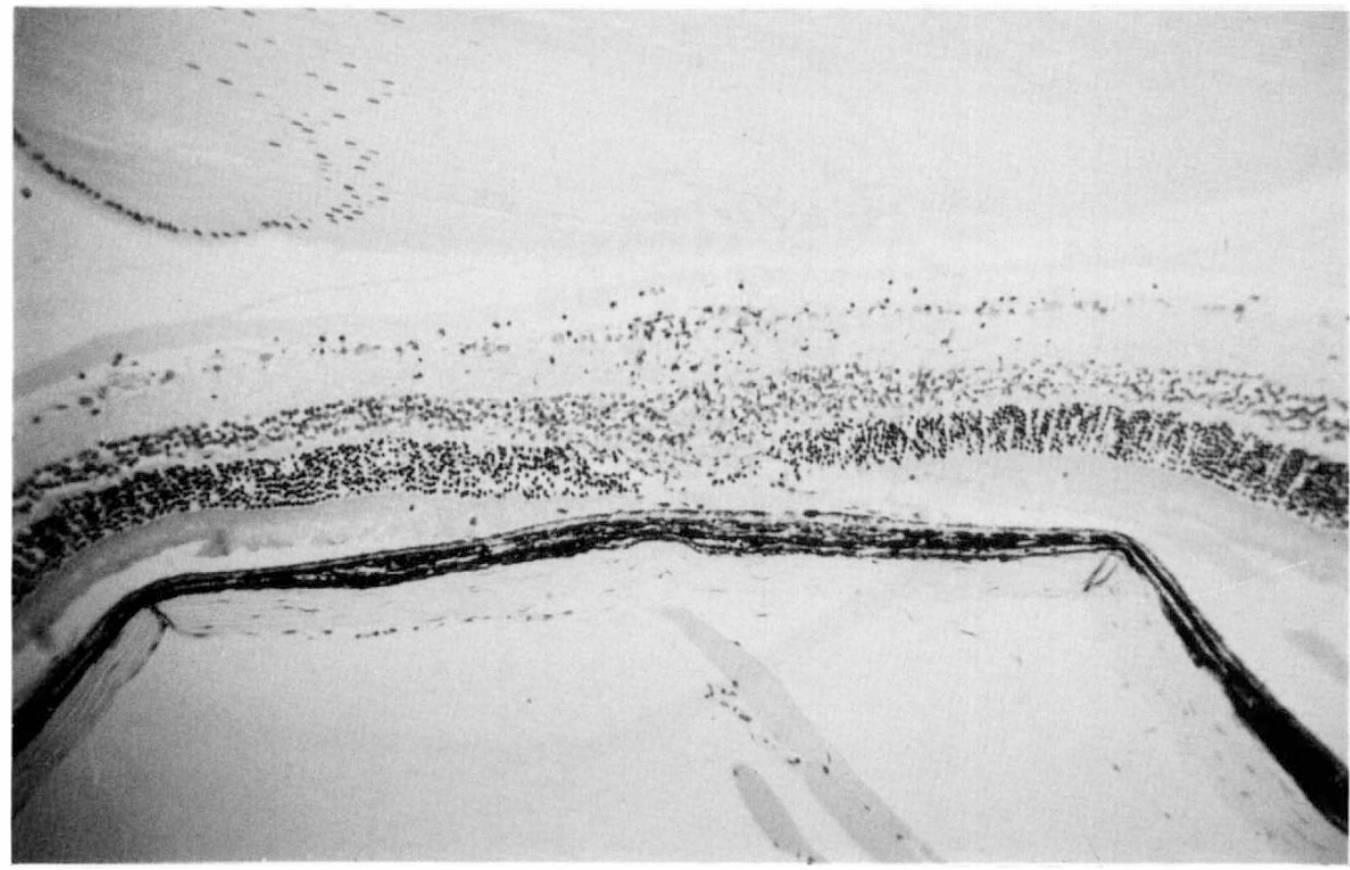

Fig. 5. Section of peripheral retina in the intermediate stage of disease. There is focal photoreceptor necrosis by mononuclear cells as shown by loss of the outer nuclear and photoreceptor layers. The retina is artefactually detached. Some fibrin and lymphocytes are seen in the vitreous $(H$. and E. $\times 200)$.

disease is characterised by a pan uveitis with obscuration of fundal details by clouding of the ocular media so that the direct observation of the evolution of clinical signs is not possible. Furthermore pathological changes in EAU are predominantly a choroiditis, with retinal destruction being associated with infiltration and thickening of the uveal structures.

In the black hooded Lister rat we have induced a self limiting form of retinal vasculitis with little anterior segment involvement so that the onset and course of the disease may be directly observed and documented. 90 per cent of our animals developed disease and this is probably due to the outbred status of this animal, in which genetically determined alterations in response between animals are present. All those animals that developed disease showed full clinical and angiographic features comparable to disease in man. However, intraretinal infiltrates are prominent in the model but uncommon in man, except in Behçet's disease. Moreover disease in this model was self limited, which is not always the case in man although relapses are frequent and may remit spontaneously (eg Behçet's hypopyon), and eventually the disease became quiescent leaving some retinal function. Serological abnormalities such as antiretinal antibodies have been detected (data not shown). However, no correlation was found between disease activity and antibody titre, in either our model or in man. ${ }^{10}$ Adoptive transfer of disease has not been attempted in this model to date but there is convincing evidence that this may be achieved using sensitised T-cells. ${ }^{11}$ Finally the black hooded Lister rat is a standard laboratory animal that has a separate retinal and choroidal circulation, unlike many other laboratory animals. Disease in the primate may be considered more analogous to man, ${ }^{12}$ but such animals are more expensive and difficult to handle, thus precluding large studies.

One significant feature of this study was that the earliest lesion observed pathologically was inflammation of the retinal vessels, rather than at the site of retinal S-antigen which is known to be found in the photoreceptors. ${ }^{7}$ These cells were eventually 
affected but the route by which inflammatory cells appeared in the photoreceptor layer was undetermined. It may be that a minimal autoimune response to retinal S-antigen presents as a retinal vasculitis, as suggested by Marak, ${ }^{13}$ so that in genetically resistant animal such as the black hooded rat this is all the response that develops.

The most striking finding in this study was that retinal vasculitis may exist in both rats and man before photoreceptor destruction with only minimal choroidal involvement. If such a stage could be identified clinically in man, possibly by electrodiagnosis, then prompt treatment may reverse the disease prior to irreversible retinal damage. Furthermore the marked lack of choroidal involvement suggests that retinal vasculitis may be primarily a retinal disease with choroidal involvement only at the later stages.

The authors wish to thank Mr. R. Dewhirst for assistance in the setting up and performance of the fluorescein angiograms and the preparation of the illustrations and Miss Josephine Lace for typing the manuscript. We are indebted to Professor A. Garner for his help and permission to use slides from the Institute of Ophthalmology. This work was supported by grants from the MRC, TFC Frost Charitable Trust, The Research (Endowments) Committee of St. Thomas's Hospital, and the Francis and Augustus Newman Foundation. M. R. Stanford is an MRC Training Fellow.

\section{References}

${ }^{1}$ Sanders MD: Retinal vasculitis: a review. J. Roy. Soc. Med. 1979; 72: 908-15.

${ }^{2}$ Dumonde DC, Kasp E, Banga JP, et al.: Autoimmune mechanisms in inflammatory eye disease. Trans. Ophthalmol. Soc. UK. 1985; 104: 232-8.

${ }^{3}$ Deschenes J, Char DH, Freeman W, et al.: Uveitis: lymphocyte subpopulation studies. Trans. Ophthalmol. Soc. UK. 1986; 105: 246-51.

${ }^{4}$ Graham E., Spalton DJ, Barnard RO, et al.: Cerebral and retinal vascular changes in systemic lupus erythematosus. Ophthalmology 1985; 92: 444-8.

${ }^{5}$ Dumonde DC, Kasp E, Graham E, et al.: Antiretinal autoimmunity and circulating immune complexes in patients with retinal vasculitis. Lancet. 1982; (ii): 787-92.

${ }^{6}$ Kasp E, Banga, JP, Sanders MD, et al.: A novel approach for the purification of human, porceine and bovine S-antigen utilizing hydrophobic absorption chromatography. In Suppl. Invest. Ophthal. Vis. Sci. (ARVO Abstracts) 1985; 26(3): $\mathrm{p} 97$.

${ }^{7}$ Gery I, Mochizuki M, Nussenblatt RB: Retinal specific antigens and Immunopathological Processes they provoke. In Progress in Retinal Research Eds. N. Osborne, J. Chader. Pergamon Press, Oxford. 1986: 75-109.

${ }^{8}$ Forrester JV, Borthwick GM, McMenamin PG: Ultrastructural pathology of S-antigen uveoretinitis. Invest. Ophth. Vis. Sci. 1985; 26: 1281-92.

${ }^{9}$ Wacker WB, Rao NA, Marak GE: Immunopathologic responses of rabbits to retina ' $S$ ' antigen. I: Phlogistic characteristics associated with antigen source and sensitising dose. Ophthalmic Res. 1981; 13: 302-11.

${ }^{10}$ Gregerson DS, Abrahams IW, Thirkill CE: Serum antibody levels of uveitis patients to bovine retinal antigens. Invest. Ophth. Vis. Sci. 1981; 21: 669-80.

${ }^{11}$ Mochizuki M, Kuwabara T, McAllister C, et al.: Adoptive transfer of experimental autoimmune uveoretinitis in rats: immunopathogenic mechanisms and histological features. Invest. Ophth. Vis. Sci. 1985; 26: 1-9.

12 Nussenblatt RB, Kuwabara T, DeMonasterio FM, et al.: S-antigen uveitis in primates: a new model for human disease. Arch. Ophthalmol. 1981; 99: 1090-92.

${ }^{13}$ Marak GE, Rao NA: Retinal ' $s$ ' antigen disease in rats. Ophthalmic Res. 1982; 14: 29-39. 